\title{
Multislice/multidetector-row computed tomography findings of a rare coronary anomaly: the first septal perforator branch originating from the left main coronary artery
}

\author{
A.G. Karadeniz' ${ }^{1}$ M.R. Sayın², S. Kul² \\ ${ }^{1}$ Department of Radiology, University of Health Sciences, Trabzon Ahi Evren Cardiovascular and Thoracic Surgery \\ Research and Application Centre, Trabzon, Turkey \\ 2Department of Cardiology, University of Health Sciences, Trabzon Ahi Evren Cardiovascular and Thoracic Surgery \\ Research and Application Centre, Trabzon, Turkey
}

[Received: 14 October 2017; Accepted: 7 December 2017]

\begin{abstract}
Multislice/multidetector-row computed tomography (MDCT) is now widely used for noninvasive assessment of coronary arteries, and it may sometimes reveal coronary anomalies. Detection of such anomalies may be relevant both during follow-up and for planning cardiac or coronary surgical/interventional procedures. These anomalies may be missed unless carefully sought. In this paper, we present the MDCT images of a first septal perforator branch originating from the left main coronary artery, which represents an extremely rare coronary anomaly. To the best of our knowledge, this is the first case in the literature where MDCT images are presented. (Folia Morphol 2018; 77, 3: 597-600)
\end{abstract}

Key words: first large septal perforator, multislice/multidetector-row computed tomography

\section{INTRODUCTION}

Anomalous coronary artery origin and course are rare occurrences but they can cause serious clinical consequences. They may cause sudden death; however, they may also remain silent throughout life. Various angiographic series reported an incidence ranging between $0.3 \%$ and $1.3 \%$ for coronary anomalies $[1,8]$. They are mostly detected incidentally. Their detection may be relevant both during follow-up and for planning cardiac or coronary surgical/interventional procedures. Hence, clinicians should be well aware of these anomalies which they may encounter in various imaging examinations.

Multislice/multidetector-row computed tomography (MDCT) has been recently started to be extensively used for noninvasive evaluation of coronary arteries. It enables to identify the origin and course as well as the clinical significance of abnormal coronary arteries detected on coronary angiography. Herein, we present the MDCT findings of an extremely rare coronary anomaly, i.e. a first septal perforator branch originating from the left main coronary artery (LMCA). As far as we know, this is the first case report in the literature where the MDCT images are provided for the reader.

\section{CASE REPORT}

A 50-year-old man presented to our outpatient clinic with chest pain. He was free of any cardiovascular risk factor other than smoking. He had normal vital signs and physical examination. His electrocardiogram (ECG) showed sinus

Address for correspondence: A. Karadeniz, MD, Sağlık Bilimleri Üniversitesi, Trabzon Ahi Evren Göğüs Kalp ve Damar Cerrahisi Sağlık Uygulama ve Araştırma Merkezi, Soğuksu Mahallesi Vatan Caddesi No: 9, Ortahisar 61040, Trabzon, Turkey, tel: +90 4622310461 , fax: +90 462 2310483, e-mail: draysegulkaradeniz@gmail.com 




Figure 1. A, B. Oblique and axial maximum intensity projection images; white arrow — first large septal perforator, arrowhead — left main; thin black arrow - circumflex; thick black arrow — left anterior descending artery.

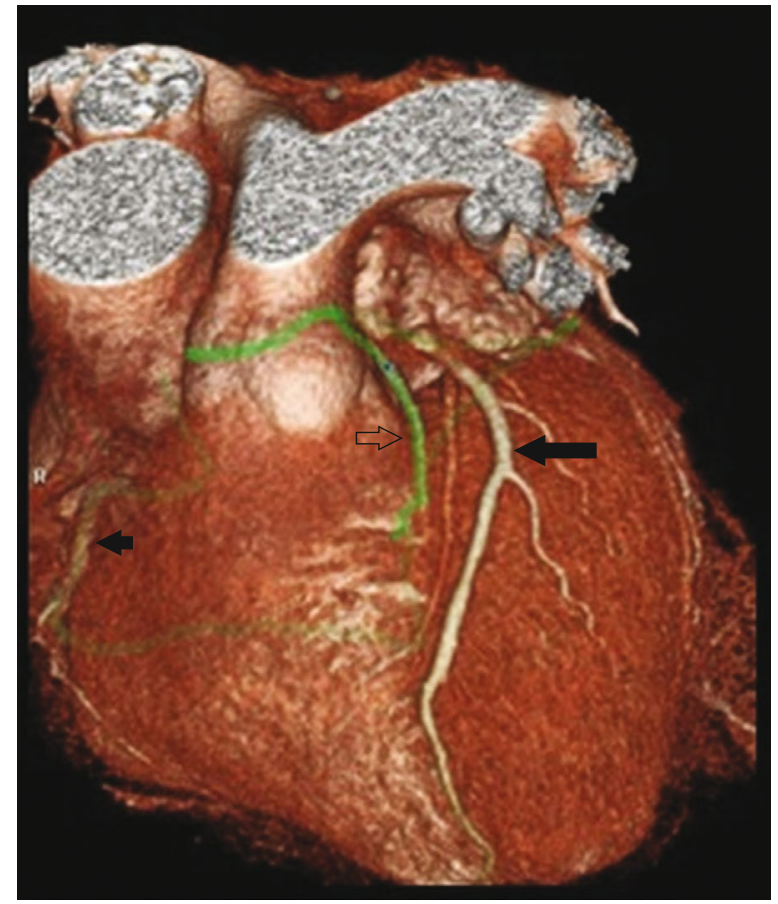

Figure 2. Volume rendered image; small black arrow — right coronary artery; big black arrow — left anterior descending artery; open arrow - first large septal perforator.

rhythm and the absence of clinically meaningful ST segment deviations. A transthoracic echocardiography revealed a normal left ventricular (LV) systolic function.
An exercise ECG test was performed for further evaluation it was not diagnostic. Than a MDCT coronary angiography was performed. No stenosis was found in any coronary artery. However, there was a well-developed coronary artery (larger first septal perforator) that originated from the mid-section of LMCA and coursed into the interventricular septum where it ended (Fig. 1). Left anterior descending artery (LAD) coursed in the anterior interventricular sulcus along the ventricular septum, and it gave off diagonal branches that coursed above the anterior wall of the LV and supplied the anterolateral aspect of the LV. However, septal perforator branches that would otherwise supply $2 / 3$ of the anterior ventricular septum could not be observed in their normal location. Furthermore, LAD did not give off septal perforator branches up to its mid-section. The axial maximum intensity projection and volume rendering technique images confirmed that the well-developed first septal branch was of LMCA origin (Fig. 2). The patient was advised to quit smoking but not administered any medical treatment.

\section{DISCUSSION}

The majority of coronary anomalies are clinically silent and they are mostly detected incidentally in coronary angiography. However, coronary anomalies 


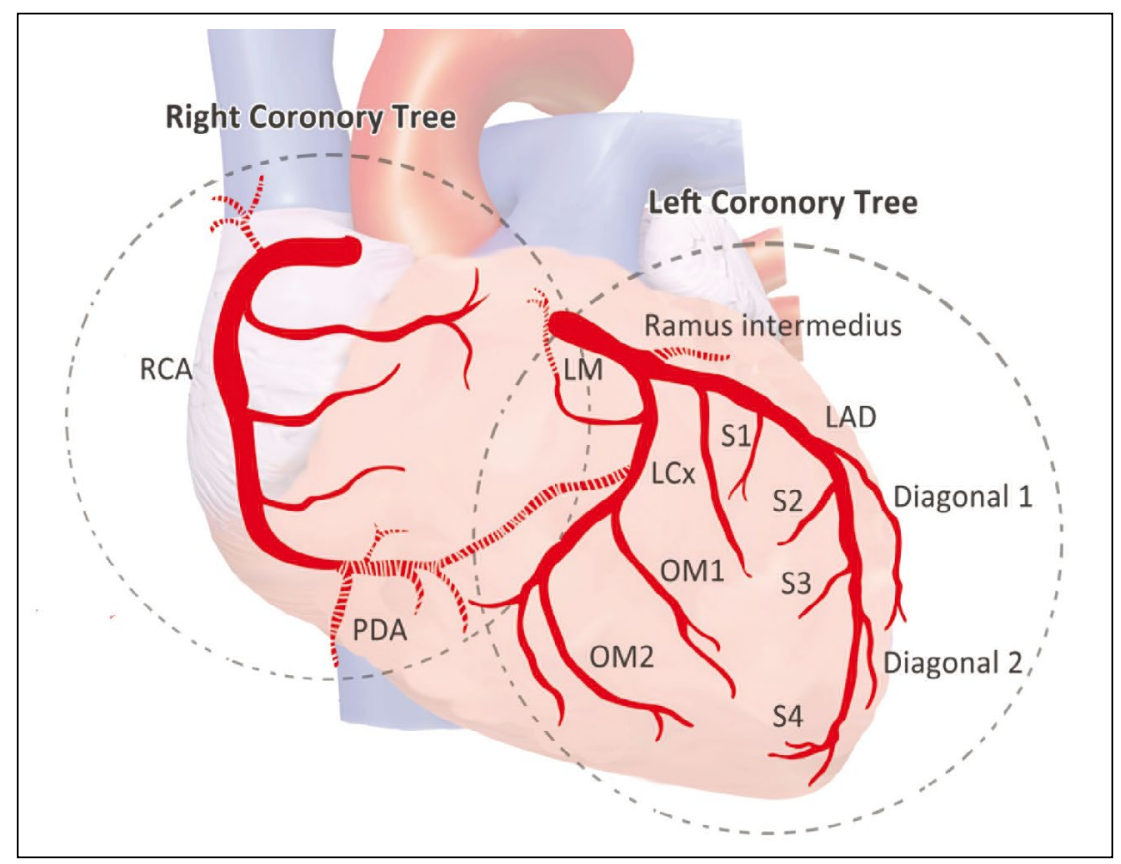

Figure 3. Schematic drawing of coronary artery branches; LCX — circumflex artery; LM — left main coronary artery; LAD — left anterior descending artery; RCA — right coronary artery; PDA — posterior descending artery; S1-S4 — septal; 0M1-2 — obtuse marginalis.

can be frequently detected by MDCT which has been recently extensively used for noninvasive assessment of coronary arteries. An accurate visualisation of the anomalous anatomy is of utmost importance for a proper management of these patients. In these instances MDCT is commonly utilised as a complimentary tool for invasive coronary angiography. Thanks to its speed and better spatial and temporal resolution characteristics, MDCT enables clinicians an accurate view of coronary arteries and their disorders. Computed tomography examines the main coronary arteries, namely LMCA, LAD, the left circumflex artery (LCX) and the right coronary artery (RCA). The anatomic relationship between coronaries is explained by the "circle and half-loop" model, where the circle is made by RCA and the LCx while the half loop consists of the LAD and the posterior descending artery [2]. LMCA normally originates from the left sinus of Valsalva and may give off LCX and LAD branches or, additionally, an intermediate ramus branch. It then usually courses downward in the anterior interventricular groove. It gives off septal perforator branches which course into the interventricular septum to supply the anterior two thirds of it (Fig. 3). A first septal arising from a non-LAD coronary vessel is a rare albeit benign clinical condition [4]. The site of origin may be aorta, RCA, LCX, diagonal artery, or obtuse marginal artery [4].
However, LMCA being the origin is an extremely rare occurrence and was not reported by the largest series of coronary anomalies ever comprising 126,995 patients [8]. Although there are several case reports about this subject in the literature, no report has ever provided MDCT images of this anomaly $[6,7]$. However, anomalous origin of a septal perforator artery has only occasionally been described in-vivo although it was identified in $12 \%$ of 427 human hearts in an autopsy study [5]. This suggests that less care is taken for angiographic assessment of septal perforators. The first anterior septal perforator artery has typically the longest $(4-6 \mathrm{~cm})$ course and the largest calibre, and it supplies a fairly big portion of the interventricular septum as well as the conduction system, namely bundle of His and the bundle branches. It also gives rise to branches that supply the atrioventricular node. It rarely provides blood supply to the anterior papillary muscle of the tricuspid valve. It is the main source of left-to-right collaterals in case of the obstruction of the RCA. Therefore, it is of clinical significance to identify its origin and course, a task which should be a routine part of a comprehensive assessment of invasive and noninvasive coronary angiograms. Furthermore, it is important to correctly identify the first septal branch among patients with hypertrophic cardiomyopathy who are scheduled for septal re- 
duction. The origins of septal perforators should be definitely scrutinised in these patients. This anomaly should also be remembered as an alternative access to chronic total occlusions when an interventional procedure is planned using the retrograde approach.

\section{CONCLUSIONS}

Coronary anomalies should be remembered in every patient undergoing invasive or noninvasive coronary angiography. It has been recently demonstrated that coronary anomalies are far more common in computed tomography than coronary angiography, which suggests a possible inaccuracy of the latter for the identification of coronary anomalies [3]. It may prove difficult to detect the origin and course of anomalous arteries by catheter angiography owing to two-dimensional images and superimposed anatomic structures. MDCT is helpful in these cases. MDCT may be of special interest for identification of the anomalous origins of septal perforators prior to septal reduction procedures among patients with hypertrophic cardiomyopathy.

\section{REFERENCES}

1. Click RL, Holmes DR, Vlietstra RE, et al. Anomalous coronary arteries: location, degree of atherosclerosis and effect on survival--a report from the Coronary Artery Surgery
Study. J Am Coll Cardiol. 1989; 13(3): 531-537, indexed in Pubmed: 2918156.

2. Daves M. Cardiac roentgenology: theloopandcircle approach. Radiology. 1970; 95(1): 157-160, doi: 10.1148/95.1.157.

3. Ghadri JR, Kazakauskaite E, Braunschweig S, et al. Congenital coronary anomalies detected by coronary computed tomography compared to invasive coronary angiography. BMC Cardiovasc Disord. 2014; 14: 81, doi: 10.1186/14712261-14-81, indexed in Pubmed: 25004927.

4. Kodama-Takahashi K, Suzuki J, Watanabe A, et al. Ischemia in the territory of the first major septal perforator branch anomalously originating from the first diagonal branch leads to a transient leftward shift of the QRS axis in the frontal plane. Circ J. 2003; 67(10): 885-888, doi: 10.1253/ circj.67.885.

5. Rodriguez FL, Robbins SL, Banasiewicz M. The descending septal artery in human, porcine, equine, ovine, bovine, and canine hearts. A postmortem angiographic study. Am Heart J. 1961; 62: 247-259, indexed in Pubmed: 13742585.

6. Sayin MR, Akpinar I, Karabag T, et al. First septal artery originating from the left main coronary artery: a rare anomaly. Herz. 2013; 38(4): 391-392, doi: 10.1007/ s00059-012-3670-5, indexed in Pubmed: 22926541.

7. Verna E, Santarone M, Boscarini M, et al. Unusual origin and course of the first septal branch of the left coronary artery: angiographic recognition. Cardiovasc Intervent Radiol. 1988; 11(3): 146-149, indexed in Pubmed: 3139296.

8. Yamanaka O, Hobbs RE. Coronary artery anomalies in 126,595 patients undergoing coronary arteriography. Cathet Cardiovasc Diagn. 1990; 21(1): 28-40, indexed in Pubmed: 2208265. 